CLINICAL STUDY

\title{
Diabetic ketoacidosis at diagnosis: role of family history and class II HLA genotypes
}

\author{
Marco Marigliano, Anita Morandi, Maddalena Maschio, Silvia Costantini, Giovanna Contreas, \\ Giuseppe D'Annunzio ${ }^{1}$, Valeria Calcaterra ${ }^{2}$ and Claudio Maffeis \\ Regional Center for Pediatric Diabetes, University of Verona, Via Bengasi, 4, 37134 Verona, Italy, ${ }^{1}$ Pediatric Clinic, Istituto Gianna Gaslini IRCCS, \\ University of Genoa, Genova, Italy and ${ }^{2}$ Department of Pediatrics, Policlinico San Matteo, Fondazione IRCCS, University of Pavia, Pavia, Italy \\ (Correspondence should be addressed to M Marigliano; Email: marco.marigliano@univrit)
}

\begin{abstract}
Objective: To explore the relationship between family history of diabetes and frequency of diabetic ketoacidosis (DKA) at diagnosis and to analyze the possible association between HLA genotypes and DKA.

Design and methods: We recruited 510 children and adolescents aged $<17$ years with type 1 diabetes (T1D) and collected information on first-degree relative (FDR) history of T1D. DKA and severe DKA were defined as blood $\mathrm{pH}<7.30$ and $<7.10$ at diabetes onset respectively. Risk categories for developing T1D were determined according to various HLA DQA1-DQB1 haplotype combination genotypes.

Results: The frequency of DKA and severe DKA at diagnosis was 34.7 and $7.2 \%$ respectively. DKA was more frequent in younger patients $(<2$ years $(60.0 \% ; P<0.001))$ and occurred less in children with at least one FDR affected by T1D (13.0 vs 37.4\%, $P<0.001)$. The logistic regression showed that age at diagnosis $(<2$ years) and increased HLA-associated risk genotypes were independent predictors of DKA $(P<0.01$, odds ratio $(\mathrm{OR})=1.068$ (95\% confidence interval $(\mathrm{CI}) 1.021-1.117) ; P<0.05$, $\mathrm{OR}=1.606(95 \% \mathrm{CI} 1.034-2.475))$. Introducing the presence of T1D in at least one FDR in the logistic model, a significant association between DKA and age at diagnosis $(<2$ years; $P<0.01, \mathrm{OR}=1.072$ (95\% CI 1.024-1.123)) and absence of FDRs with T1D $(P=0.001$, OR $=4.287$ (95\% CI $1.770-$ $10.383)$ ) was found, but no more with increased HLA-associated risk genotype $(P=0.06, \mathrm{OR}=1.550$ (95\% CI 0.992-2.423)).

Conclusions: HLA-associated high-risk genotypes are associated with a high chance of presenting DKA at diabetes onset. However, having at least one FDR with T1D reduced the risk of DKA regardless of HLA genotype.
\end{abstract}

European Journal of Endocrinology 168 107-111

\section{Introduction}

Diabetic ketoacidosis (DKA) is the leading cause of morbidity and mortality in children with type 1 diabetes (T1D). The frequency of DKA at T1D onset has a wide geographic variation, and rates inversely correlate with the regional incidence of the disease (1). DKA at onset of T1D is more common in younger children $(<4$ years of age), children without a first-degree relative (FDR) with T1D, and those from families of lower socioeconomic status (2). Rising standards of medical information and greater awareness concurrent with an increase in incidence have modified the clinical presentation at onset of childhood T1D in Europe $(3,4,5)$.

Specific HLA class II gene alleles at the HLA-DRB1, DQA1, and DQB1 loci are the major genetic determinants of susceptibility and resistance to T1D. In Caucasian populations, the highest risk for developing the disease has been associated with the DR3-DQA1*0501-DQB1*0201/DR4-DQA1*0301DQB $1 * 0302$ genotype, whereas the strongest negative association has been found with the DRB1*1501DQA1*0102-DQB1*0602 genotype (6). At present, little is known about the association between HLA class II genotypes and DKA risk at diagnosis of T1D, although this might be clinically relevant in FDRs of subjects affected by T1D (7). Recently, a Finnish study showed that children with a strong/moderate risk of T1D HLA genotypes had lower frequency of DKA and severe DKA than subjects with other genotypes (8). On the basis of the variations in the distribution of particular DQ genotypes that confer a high risk of T1D in the general population in Europe, it appears interesting to analyze a population with a lower incidence of T1D and a different genetic background, such as that of Italians (9). 
The aim of this study was to explore the relationship between family history of diabetes and frequency of DKA at diagnosis and to analyze a possible association between HLA genotypes and DKA rate in a sample of Italian children with T1D.

\section{Materials and methods}

The study cohort comprised 510 children and adolescents aged $<17$ years who had been diagnosed with T1D in three Pediatric Units of northern Italy (Verona, Pavia, and Genoa) between 2000 and 2011. The protocol was in accordance with the 1975 Declaration of Helsinki as revised in 2008 and approved by the Committee of Public Health Care Authority of Verona. Informed consent was obtained from all the children and their parents.

Information on family history (mother, father, siblings, and grandparents) of type 1 and type 2 diabetes was collected by interviewing the parents of participating children. Definition of T1D was based on diagnosis at a young age, presence of $\beta$-cell autoantibodies, and/or persistent requirement of exogenous insulin from the time of diagnosis. Gestational diabetes, monogenic diabetes, or unclassified diabetes was not included in the analysis. DKA was defined as blood $\mathrm{pH}<7.30$ and severe DKA as $\mathrm{pH}<7.10$ at diabetes onset (2).

Risk categories for developing T1D (strongly increased, moderately/slightly increased, neutral, or decreased) were determined according to various HLA DQA1-DQB1 haplotype combination genotypes. The strongly increased group included heterozygosity for the two risk-associated haplotypes DQA1*0501DQB $1 * 0201$ and DQA $1 * 0301 / 2 / 3-D Q B 1 * 0302$. The moderately/slightly increased group included homozygosity for the two risk-associated haplotypes or one risk-associated haplotype combined with a slightly increased risk/neutral/protective haplotype. The neutral risk group is characterized by having a genotype with no risk or protective haplotypes. The decreased risk group is characterized by having combinations of two protective haplotypes or a protective haplotype associated with a neutral one. DKA frequencies were compared between the four HLA-associated risk groups and with family history of diabetes.
Data were analyzed using SPSS version 19.0 software (SPSS, Chicago, IL, USA). Data are shown as mean and S.D. Differences between the two groups were assessed by Student's $t$-test after checking for normal distribution. The Mann-Whitney $U$ test was used for unequally distributed variables. Distributions were analyzed by cross-tabulation and $\chi^{2}$ statistics. A logistic regression analysis was also done to determine whether the frequency of DKA was related to age, sex, HLA genotype, and T1D in FDRs; the results are reported as odds ratio $(\mathrm{OR})$ and $95 \%$ confidence interval $(\mathrm{CI})$. A $P$ value $<0.05$ was considered statistically significant.

\section{Results}

Five hundred and ten (268 boys, 52.5\%) subjects with T1D were recruited. The mean age at diagnosis was 7.8 (range 0.8-16.0) years. The frequency of DKA $(\mathrm{pH}<7.3)$ at diagnosis was $34.7 \%(177 / 510)$ and the frequency of severe DKA $(\mathrm{pH}<7.1)$ at diagnosis was $7.2 \%(37 / 510)$. DKA was reported in $31.7 \%$ of boys $(85 / 268)$ and $38.0 \%$ of girls $(92 / 242)$; severe DKA was reported in $6.3 \%$ of boys $(17 / 268)$ and $8.3 \%$ of girls $(20 / 242)$. DKA was more frequent in younger patients (age $<2$ years) with a frequency almost twice that of the other age groups $(2-5,5-10$, and $>10$ years $)$ $(60.0 \%$ vs $29.5 \%, 34.3 \%$, and $27.6 \% ; P<0.001)$ (Table 1). In the total sample, the diabetes status of the FDRs was known, and in 9.4\% (48 out of 510) of the patients, at least one FDR was affected by T1D; in $46(95.8 \%)$ of these families, there was only one FDR with T1D and two families $(4.2 \%)$ had at least two family members with T1D. DKA occurred with less frequency in children with at least one FDR affected by T1D compared with children with no FDRs affected by T1D $(13.0$ vs $37.4 \%, P<0.001)$. There was no particular association between DKA frequency and grandparents with T1D or T2D (data not shown).

An HLA-associated risk was defined in all the enrolled children. No statistically significant differences were found in the HLA genotype risk category distribution between boys and girls and among different age groups $(<2,2-5,5-10$, and $>10$ years). Subjects with strongly increased or moderately/slightly increased HLA-associated risk genotypes were more likely to

Table 1 The number of subjects $(n)$ and the frequencies $(\%)$ of total enrolled patients with $\mathrm{DKA}(\mathrm{pH}<7.3)$ or severe $\mathrm{DKA}(\mathrm{pH}<7.1)$ at T1D onset according to age groups and gender.

\begin{tabular}{|c|c|c|c|c|c|c|c|c|c|}
\hline \multirow{2}{*}{$\begin{array}{l}\text { Age at diagnosis } \\
\text { (years) }\end{array}$} & \multicolumn{3}{|c|}{$\begin{array}{c}\text { Total subjects } \\
n \text { and frequencies (\%) }\end{array}$} & \multicolumn{3}{|c|}{$\begin{array}{c}\text { DKA }(\mathrm{pH}<7.3) \\
n \text { and frequencies }(\%)\end{array}$} & \multicolumn{3}{|c|}{$\begin{array}{l}\text { Severe DKA }(\mathrm{pH}<7.1) \\
\boldsymbol{n} \text { and frequencies }(\%)\end{array}$} \\
\hline & Boys & Girls & Total & Boys & Girls & Total & Boys & Girls & Total \\
\hline$<2$ & $31(11.6)$ & $34(14.1)$ & $65(12.7)$ & $18(58.0)$ & $21(61.8)$ & $39(60.0)$ & $3(9.7)$ & $6(17.6)$ & $9(13.8)$ \\
\hline $2-5$ & $61(22.8)$ & 44 (18.2) & 105 & $16(26.2)$ & 15 & 31 & $1(1.6)$ & $2(4.5)$ & $3(2.8)$ \\
\hline $5-10$ & $93(34.7)$ & $102(42.1)$ & $195(38.3)$ & $32(34.4)$ & 35 (34.3) & 67 (34.3) & $9(9.7)$ & $8(7.8)$ & 17 (8.7) \\
\hline$>10$ & $83(30.9)$ & $62(25.6)$ & $145(28.4)$ & 19 (22.9) & 21 (33.8) & $40(27.6)$ & $4(4.8)$ & $4(6.4)$ & $8(5.5)$ \\
\hline Total & $268(52.5)$ & $242(47.5)$ & $510(100)^{\prime}$ & $85(31.7)$ & $92(38.0)$ & $177(34.7)$ & 17 (6.3) & 20 (8.3) & $37(7.2)$ \\
\hline
\end{tabular}


Table 2 The number of subjects $(n)$ and the frequencies (\%) in different $\mathrm{HLA}$ risk categories of enrolled patients with DKA or severe DKA. Linear-by-linear association analysis was used to compare DKA frequencies between various risk groups.

\begin{tabular}{lcc}
$\begin{array}{l}\text { HLA-associated risk groups } \\
\boldsymbol{n} \text { and frequencies (\%) }\end{array}$ & DKA (\%) & Severe DKA (\%) \\
\hline $\begin{array}{l}\text { Strongly increased } \\
\text { risk: } 106(20.8)\end{array}$ & $47(26.6)$ & $12(32.4)$ \\
$\begin{array}{l}\text { Moderately increased } \\
\text { risk: } 300(58.8)\end{array}$ & $99(55.9)$ & $18(48.7)$ \\
Neutral risk: $94(18.4)$ & $27(15.2)$ & $5(13.5)$ \\
Decreased risk: $10(2.0)$ & $4(2.3)$ & $2(5.4)$ \\
Total: $510(100.0)$ & $177(100.0)$ & $37(100.0)$ \\
$P$ value & 0.096 & 0.100 \\
\hline
\end{tabular}

present DKA at T1D onset compared with subjects with a neutral or decreased HLA-associated risk genotype (82.5 vs $17.5 \% ; P<0.001$ ) (Table 2 ).

Children younger than 2 years (those with the highest risk of developing DKA) had increased HLA-associated risk genotypes in almost $90 \%$ of cases (89.5 vs $10.5 \% ; P<0.001$ ). Neutral/decreased HLAassociated risk genotypes and increased HLA-associated risk genotypes had the same frequency in patients with at least one FDR affected by T1D compared with children with no FDR with T1D (80.1 vs $78.3 \% ; 19.9$ vs $21.7 \%$; $P=\mathrm{NS}$ ).

The logistic regression model was applied to identify variables associated with DKA, including age at diagnosis, gender, HLA DQB1 risk categories, and the presence of T1D in FDRs. Age at diagnosis $(<2$ years of age) and HLA risk categories (increased HLA-associated risk genotypes) were identified as independent predictors of DKA $(P<0.01, \mathrm{OR}=1.068(95 \% \mathrm{CI} 1.021-$ $1.117) ; P<0.05, \mathrm{OR}=1.606(95 \%$ CI $1.034-2.475)$ respectively). When the presence of T1D in at least one FDR was introduced in the logistic model, a significant association between DKA and age at diagnosis $(<2$ years of age; $P<0.01, \mathrm{OR}=1.072$ $(95 \%$ CI $1.024-1.123))$ and absence of FDRs with T1D $(P=0.001, \mathrm{OR}=4.287(95 \%$ CI $1.770-10.383))$ was found, but having an increased HLA-associated risk genotype was no longer significant $(P=0.06$, $\mathrm{OR}=1.550(95 \%$ CI $0.992-2.423))($ Table 3$)$.

\section{Discussion}

To the best of our knowledge, this is the first populationbased analysis of Italian children and adolescents aged $<17$ years diagnosed with T1D, in which the association between HLA DQB1 genotypes and the presence of DKA at diabetes onset was explored. From our data, a very young age was consistently associated with a higher risk of DKA at diagnosis. This increased risk was most noticeable in children under 2 years with a frequency almost twice that of the other age groups
$(2-5,5-10$, and $>10$ years). There are probably several reasons for this. The most likely explanation is the less frequent suspicion of diabetes in younger children as well as the classical symptoms of diabetes that are difficult to distinguish from other acute illnesses at this age (10). Dehydration and acidosis also develop more quickly in young children and the mechanisms of metabolic compensation are less efficient $(10,11)$. Moreover, $\beta$-cell destruction may be more aggressive in children under 2 years at onset of diabetes, and their endogenous insulin secretory capacity worsens faster than in older children $(10,12)$.

Our study confirms that the presence of T1D in an FDR protects the child from DKA at diagnosis, as was previously reported in a Finnish study and in a recent systematic review $(8,10)$. Interestingly, the protective effect of family history of diabetes seems to be more important than the presence of strongly increased or moderately/slightly increased risk HLA DQB1 genotypes. In the population with no FDRs with T1D, strongly increased or moderately/slightly increased risk HLA DQB1 genotypes are directly correlated with DKA at T1D onset, as shown by the logistic regression analysis. Frequency of DKA at onset was lower (13.4\%) in children with at least one FDR affected by T1D, whereas in children with no FDRs with T1D, we found an almost threefold frequency of DKA (37.4\%). Similar observations have been reported in previous studies (3, 8). The reasons for the reduced frequency of DKA in children with at least one FDR with T1D are comprehensible. In those families, the signs and symptoms of diabetes onset are well known, equipment for blood glucose self-monitoring is usually available, and parents with experience will more likely contact a doctor who can prescribe treatment promptly $(10,13)$. No statistical correlations were found between the presence of type 2 diabetes in FDR of children with T1D and DKA at diabetes onset.

Several studies have reported the effect of family history of diabetes on presentation with DKA at T1D onset. Although having a FDR with T1D decreased the frequency of DKA in three studies $(14,15,16)$, it did not predict a diagnosis of new onset of diabetes before progression to DKA in a US study after adjustment for sex, age, primary or secondary care diagnosis, and

Table 3 The number of subjects $(n)$ and the frequencies (\%) of patients with DKA $(\mathrm{pH}<7.3)$ or not DKA $(\mathrm{pH} \geq 7.3)$ at T1D onset according to HLA-associated risk groups and the presence of FDRs with $(+)$ or without $(-)$ T1D.

\begin{tabular}{lccccc}
\hline & \multicolumn{2}{c}{$\begin{array}{c}\text { Strongly/moderately } \\
\text { increased risk HLA } \\
\text { genotypes }(\%)\end{array}$} & & \multicolumn{2}{c}{$\begin{array}{c}\text { Neutral/decreased risk } \\
\text { HLA genotypes (\%) }\end{array}$} \\
\cline { 2 - 3 } $\begin{array}{l}\text { Presence } \\
\text { of DKA }\end{array}$ & FDRs + & FDRs - & & FDRs + & FDRs - \\
\hline $\mathrm{pH}<7.3$ & $2(0.5)$ & $144(35.5)$ & & $3(2.9)$ & $28(26.9)$ \\
$\mathrm{pH} \geq 7.3$ & $36(8.6)$ & $224(55.4)$ & & $9(8.7)$ & $64(61.5)$ \\
\hline
\end{tabular}


duration of symptoms (17). A German study adjusted for sex, age, and social status also failed to show a significant association with a family history of either type 1 or type 2 diabetes in siblings, parents, or grandparents (18). In a recent study, Hekkala et al. (8) showed that children with a strong/moderate risk of T1D HLA genotypes had a lower frequency of DKA and severe DKA than subjects with other genotypes. On the contrary, in our study, subjects with strongly increased or moderately/slightly increased HLA-associated risk genotypes were more likely to present DKA at T1D onset, compared with subjects with neutral or decreased HLA-associated risk genotypes (82.5 vs $17.5 \%$; $P<0.001)$. The reasons for this discrepancy could likely be found in a greater awareness of T1D and its symptoms in the Finnish population on the basis of the higher prevalence of this disease in Finland than in Italy and of the effect of the ongoing prospective T1D Prediction and Prevention (DIPP) study in Finland. Through this study, all the newborn infants born in three university hospitals were screened for HLAconferred susceptibility to T1D, and those with HLA genotypes conferring high or moderate disease risk were monitored from their day of birth with periodic determination of T1D-associated autoantibodies $(3,19)$. T1D was usually diagnosed in those patients at a very early stage and this contributes to lowering the risk of DKA among these children. In Italy (and in other European countries), there is no national HLAconferred susceptibility to T1D screening program because of the lower incidence of the disease and the high cost of this kind of screening program. Consequently, it would be very difficult to identify T1D onset at a very early stage without an adequate knowledge of typical signs and symptoms of T1D. In this study, we stress the importance and protective effect of having at least one FDR with T1D in reducing DKA incidence at diabetes onset. Logistic regression analysis showed that children $<2$ years are the age category with the highest risk of presenting DKA at T1D onset even if OR values are not so remarkable; the presence of a parent or sibling with T1D had a protective effect that is more important even than the HLA-associated risk genotypes.

This study has certain limitations: i) our study cohort cannot be considered representative of the entire national population. However, the prevalence and incidence of T1D are similar in the north and south of the country, excluding Sardinia; ii) the lack of data regarding socioeconomic status of patients and their families, possible confounding factors; and iii) differences in DKA incidence could be affected by different degrees of medical awareness about T1D and DKA treatment protocols used in different pediatric units. However, a national consensus on clinical management of T1D at onset is available (20). The strengths of this study were: i) the novelty of the study, never having been performed in a European population with a relatively low incidence of T1D, such as Italy; and ii) the potential clinical implications of the results, suggesting the importance of improving awareness of T1D and its symptoms at onset in the general population.

In conclusion, this study shows that HLA-associated high-risk genotypes and the very young age at T1D diagnosis $(<2$ years $)$ are associated with a high chance of presenting DKA at diabetes onset. However, family history characterized by having at least one FDR with T1D reduced the risk of DKA regardless of HLA genotype.

\section{Declaration of interest}

The authors declare that there is no conflict of interest that could be perceived as prejudicing the impartiality of the research reported.

\section{Funding}

This research did not receive any specific grant from any funding agency in the public, commercial or not-for-profit sector.

\section{Author contribution statement}

M Marigliano contributed to the design of the study, recruited the children, provided data input, collaborated on the statistical analysis, and writing of the manuscript; A Morandi collaborated on the statistical analysis and writing of the manuscript; M Maschio collaborated on the statistical analysis and writing and editing the manuscript; S Costantini provided data input and analysis and collaborated on the discussion of the paper; G Contreas recruited the children, provided data input, and collaborated on the statistical analysis; G D'Annunzio recruited the children and collaborated on data input and contributed to discussion; $\mathrm{V}$ Calcaterra recruited the children, collaborated on data input and contributed to discussion; $\mathrm{C}$ Maffeis designed the study, submitted the study to the Ethics Committee of the University, performed the statistical analysis, and wrote the manuscript.

\section{References}

1 Levy-Marchal C, Patterson CC \& Green A. Geographical variation of presentation at diagnosis of type I diabetes in children: the EURODIAB Study. Diabetologia 200144 (Suppl 3) B75-B80. (doi:10.1007/PL00002958)

2 Wolfsdorf J, Craig ME, Daneman D, Dunger D, Edge J, Lee W, Rosenbloom A, Sperling M \& Hanas R. Diabetic ketoacidosis in children and adolescents with diabetes. Pediatric Diabetes 2009 10 (Suppl 12) 118-133. (doi:10.1111/j.1399-5448.2009. 00569.x)

3 Rewers A, Klingensmith G, Davis C, Petitti DB, Pihoker C, Rodriquez B, Schwartz ID, Imperatore G, Williams D, Dolan LM et al. Presence of diabetic ketoacidosis at diagnosis of diabetes mellitus in youth: the Search for Diabetes in Youth Study. Pediatrics 2008121 e1258-e1266. (doi:10.1542/peds.2007-1105)

4 Vanelli M, Chiari G, Ghizzoni L, Costi G, Giacalone T \& Chiarelli F. Effectiveness of a prevention program for diabetic ketoacidosis in children. An 8-year study in schools and private practices. Diabetes Care 199922 7-9. (doi:10.2337/diacare.22.1.7)

5 Hekkala A, Knip M \& Veijola R. Ketoacidosis at diagnosis of T1D in children in northern Finland: temporal changes over 20 years. Diabetes Care 200730 861-866. (doi:10.2337/dc06-2281)

6 Hermann R, Turpeinen H, Laine AP, Veijola R, Knip M, Simell O, Sipila I, Akerblom HK \& Ilonen J. HLA DR-DQ-encoded genetic 
determinants of childhood-onset T1D in Finland: an analysis of 622 nuclear families. Tissue Antigens 200362 162-169. (doi:10.1034/j.1399-0039.2003.00071.x)

7 Salardi S, Zucchini S, Cicognani A, Corbelli E, Santoni R, Ragni L, Elleri D \& Cacciari E. The severity of clinical presentation of T1D in children does not significantly influence the pattern of residual b-cell function and long-term metabolic control. Pediatric Diabetes 20034 4-9. (doi:10.1034/j.1399-5448.2003. 00028.x)

8 Hekkala A, Ilonen J, Knip M, Veijola R \& the Finnish Paediatric Diabetes Register. Family history of diabetes and distribution of class II HLA genotypes in children with newly diagnosed T1D: effect on diabetic ketoacidosis. European Journal of Endocrinology 2011165 813-817. (doi:10.1530/EJE-11-0376)

9 Rønningen KS, Keiding N, Green A \& EURODIAB ACE Study Group. Correlations between the incidence of childhood-onset type I diabetes in Europe and HLA genotypes. Diabetologia $2001 \mathbf{4 4}$ (Suppl 3) B51-B59. (doi:10.1007/PL00002955)

10 Usher-Smith JA, Thompson MJ, Sharp SJ \& Walter FM. Factors associated with the presence of diabetic ketoacidosis at diagnosis of diabetes in children and young adults: a systematic review. BMJ 2011343 d4092. (doi:10.1136/bmj.d4092)

11 Samuelsson U \& Stenhammar L. Clinical characteristics at onset of T1D in children diagnosed between 1977 and 2001 in the south-east region of Sweden. Diabetes Research and Clinical Practice 200568 49-55. (doi:10.1016/j.diabres.2004.08.002)

12 Komulainen J, Kulmala P, Savola K, Lounamaa R, Ilonen J, Reijonen H, Knip M \& Akerblom HK. Clinical, autoimmune, and genetic characteristics of very young children with T1D. Childhood Diabetes in Finland (DiMe) Study Group. Diabetes Care 199922 1950-1955. (doi:10.2337/diacare.22.12.1950)

13 Komulainen J, Knip M, Sabbah E, Vähäsalo P, Lounamaa R, Akerblom HK, Reijonen H \& Ilonen J. Autoimmune and clinical characteristics of type I diabetes in children with different genetic risk loads defined by HLA-DQB1 alleles. Childhood Diabetes in Finland Study Group. Clinical Science 199894 263-269.
14 Pinkney JH, Bingley PJ, Sawtell PA, Dunger DB \& Gale EAM. Presentation and progress of childhood diabetes mellitus: a prospective population-based study. Diabetologia 199437 70-74. (doi:10.1007/BF00428780)

15 Veijola R, Reijonen H, Vähäsalo P, Sabbah E, Kulmala P, Ilonen J, Akerblom HK \& Knip M. HLA-DQB1-defined genetic susceptibility, beta cell autoimmunity, and metabolic characteristics in familial and nonfamilial insulin-dependent diabetes mellitus. Journal of Clinical Investigation 199698 2489-2495. (doi:10.1172/JCI119067)

16 Abdul-Rasoul M, Al-Mahdi M, Al-Qattan H, Al-Tarkait N, Alkhouly M, Al-Safi R, Al-Shawaf F \& Mahmoud H. Ketoacidosis at presentation of T1D in children in Kuwait: frequency and clinical characteristics. Pediatric Diabetes 201011 351-356. (doi:10.1111/j.1399-5448.2009.00600.x)

17 Quinn M, Fleischman A, Rosner B, Nigrin DJ \& Wolfsdorf JI. Characteristics at diagnosis of T1D in children younger than 6 years. Journal of Pediatrics 2006148 366-371. (doi:10.1016/ j.jpeds.2005.10.029)

18 Rosenbauer J, Icks A \& Giani G. Clinical characteristics and predictors of severe ketoacidosis at onset of T1D mellitus in children in a North Rhine-Westphalian region, Germany. Journal of Pediatric Endocrinology and Metabolism 200215 1137-1145.

19 Kupila A, Muona P, Simell T, Arvilommi P, Savolainen H, Hamalainen AM, Korhonen S, Kimpimaki T, Sjoroos M, Ilonen J et al. Feasibility of genetic and immunological prediction of type I diabetes in a population-based birth cohort. Diabetologia 2001 44 290-297. (doi:10.1007/s001250051616)

20 Gentile S, Cavallo Perin P, Giorda CB and Riccardi G. Associazione Medici Diabetologi (AMD) - Società Italiana di Diabetologia (SID). Standard italiani per la cura del diabete mellito 2009-2010; Torino: Ed. Infomedica, 2010. (available at http://www.siditalia.it).

Received 21 June 2012

Revised version received 30 August 2012

Accepted 12 October 2012 